\title{
Cheap talk, Efficiency and Egalitarian Cost Sharing In Joint Projects
}

\author{
Murali Agastya \\ (m.agastya@econ.usyd.edu.au)
}

Economics Discipline, SEPS

Merewether H04

University of Sydney

Sydney NSW 2006, AUSTRALIA

\author{
Flavio Menezes \\ (flavio.menezes@anu.edu.au)
}

Australian Centre of Regulatory Economics

Copland Building (No. 26)

Australian National University

Canberra ACT 0200, AUSTRALIA

\author{
Kunal Sengupta \\ (k.sengupta@econ.usyd.edu.au) \\ Economics Discipline, SEPS \\ Merewether H04 \\ University of Sydney \\ Sydney NSW 2006, AUSTRALIA
}

First Draft: May 31, 2003, This version 26 August, 2005

\begin{abstract}
Rarely, if ever, do parties contemplating a joint project commit resources without engaging in non-binding discussions about who does what. To examine the role of such discussions, we model the investment decision as a voluntary contribution game where each player is privately informed of her benefit from project's completion. Efficiency of every equilibrium of this game is improved when a prior stage of communication is allowed. Interestingly, this improvement can be achieved in "simple equilibria" where a player simply announces whether (not how much) she plans to contribute. Analogous results hold in terms of the probability of completion instead of efficiency. When players' types are identically distributed, the superior utilitarian welfare maximizing equilibrium has an egalitarian feature - whenever the project is completed, each party contributes exactly half the cost, independent of private information
\end{abstract}


Rarely, if ever, do parties contemplating a joint project commit resources without engaging in non-binding discussions on who does what. As one might expect, such discussions are more prominent when contemplating large undertakings where no one member has an incentive to complete the project on her own and spending her own resources without some assurance from the others would be too risky. For instance, the International Thermonuclear Energy Reactor (ITER) program to demonstrate the scientific and technological feasibility of fusion energy began as an initiative between the US and Russia as early as 1985. It took over three years before formal collaboration began. Serious negotiations for a site for the reactor to reduce the cost of implementation of the program did not take place until 2003. Similarly, the USCAR, a consortium representing a joint research venture between the Big Three auto makers did not take shape until 1992, although the initiative goes back to the period following the Cooperative Research Act (1984) by the U.S. Congress ${ }^{1}$

In this paper, we model the investment choices of two players who seek to undertake a joint project as a voluntary contributions game familiar from the literature on the private provision of discrete public goods. The two players simultaneously choose how much to invest in the project, with the knowledge that any investment is foregone even if the project is not completed. The project is completed only if the sum of their investments is at least the fixed (and commonly known) cost $k$. The incremental benefit from a project's completion to a player is however her private information. Our focus throughout is on situations where no one player has an incentive to complete the project on her own.

Section 2 contains a fairly complete analysis of this game. Proposition 1 presents a simple necessary and sufficient condition for a positive (equilibrium) probability of the project's completion when the priors have interval supports. The condition itself captures the intuition that for the completion of the project, the sum of interim benefits of the lowest types of the two players that might contribute a positive amount must cover the cost of the project. Proposition 2 uses this to show that for concave priors in particular, either there is an equilibrium in which the project is completed with probability one or the project is never completed. We view this exhaustive analysis of the contribution game as adding to a large literature on the private provision of public goods using a voluntary contribution schem 2 . It also sets the stage to study the implications of cheap talk.

In Section 3, we augment a prior stage of communication to the above game. The communication scheme we consider is very simple - players simultaneously communicate only whether they plan to make a contribution (" $y$ ") or not (" $n$ "). Further, we restrict attention to "simple equilibria". In a simple equilibrium, denoted by $\mathcal{S}(x)$, there is a threshold $x$. Player 1 and Player 2 first announce $y$ only if their respective values for the project are at least $x$ and $k-x$. Unanimous announcements of $y$ are followed up by a contribution of $x$ by Player 1 and $k-x$ by Player 2 and the project is completed. Otherwise no contributions are made.

\footnotetext{
${ }^{1}$ See http://www.ofes.fusion.doe.gov/iter.html and http://www.uscar.org/uscar/history.htm for information on ITER project and USCAR respectively.

${ }^{2}$ An exception is Menezes et al. (2001) which studies other mechanisms besides the contribution mechanism. They do not consider the role of cheap talk. Regardless, for the two player contribution game, our informational assumptions are more general and the results sharper.
} 
Proposition 4 shows that every equilibrium of the contribution game is Pareto dominated in an interim and an ex-ante sense by some simple equilibrium despite the rather coarse communication the latter. If instead of the Pareto criterion, a scalar welfare measure is used to rank equilibria, such as the ex-ante probability of the project's completion or the players' aggregate welfare, then all the equilibria of the contribution game can be uniformly dominated by a single simple equilibrium. (See Proposition 3 and Proposition 5). Furthermore, if types are identically distributed according to a regular distribution, it turns out that the most efficient simple equilibrium (according to the utilitarian social choice rule or the probability of completion), is the one where both parties share the cost equally. The "best" equilibrium in this case is curiously egalitarian.

An intuition for why communication results in superior outcomes can be gained by entertaining, for the moment, the existence of an equilibrium of the original game of the following kind: Player 1 (Player 2) of a type above a threshold $\hat{v}_{1}\left(\hat{v}_{2}\right)$ contributes an amount $x(k-x)$ and other types do not contribute. The project is completed only in the states $\left(v_{1}, v_{2}\right)$ such that $v_{1} \geq \hat{v}_{1}$ and $v_{2} \geq \hat{v}_{2}$. The total contribution from the marginal types is $k$. Therefore, the total expected benefit of these types must be as high as $k$ and since no one player completes the project on her own, $\hat{v}_{1}+\hat{v}_{2}>k$. On the other hand, in the simple equilibrium $\mathcal{S}\left(\hat{v}_{2}\right)$, the project is completed in the additional states $\left(v_{1}, v_{2}\right)$ such that $k-\hat{v}_{2} \leq v_{1} \leq \hat{v}_{1}$ and $v_{2} \geq \hat{v}_{2}$. This leads to an increased probability of completion in an ex-post efficient region and results in superior efficiency. Lemma 2 provides the key ingredient in the above argument for arbitrary equilibria of the contribution game, namely that the marginal valuations that make a positive contribution sum to greater than $k$. This lies at the heart of our efficiency results.

Although the focus of the paper is on how limited communication can help, in Section 4.2 we show that any ex-ante efficient mechanism among those that satisfy IC and ex-post IR can be implemented as full communication equilibria if players announce their types. Remaining subsections of Section 4 discuss other aspects of our work.

This paper studies the implications of adding a non-binding communication stage to an economic game. Other such contributions include Crawford and Sobel (1982), Farrell and Gibbons (1989), Forges (1990), Baliga and Sjostrom (2003), Doraszelski et al. (2003) and Matthews and Postlewaite (1989). The reader is directed to Farrell and Rabin (1996) or Baliga and Morris (2002) and the references therein for a further discussion of the role of unmediated communication.

In Farrell and Gibbons (1989) and Matthews and Postlewaite $(1989)$ the underlying economic game is a bilateral trade of a private good through a $\kappa$-double auction. Whereas we demonstrate that communication is always welfare enhancing for the contribution game, it is not necessarily so for the double auction. This is clear since it is known that, albeit under special assumptions on the priors, the ex-ante efficient payoff frontier among all bilateral trading mechanisms that satisfy IC and ex-post IR can be implemented through equilibria of $\kappa$-double auctions for varying values of $\kappa$. (See Satterthwaite and Williams (1989)). In Baliga and Sjostrom (2003), unlike here, the ex-post efficient outcome is independent of players' types, In Doraszelski et al. (2003), the ex-post efficient outcomes depend on aggregate uncertainty which imply correlated utilities.

To summarize, the contribution game is unique in that allowing exchange of binary messages is enough for greater efficiency despite the fact that it has a continuum of types and 
actions.

\section{The Contribution Game}

A joint project between two partners requires a total investment of $k>0$ to complete. The players simultaneously choose how much to contribute. For $i=1,2$, if Player $i$ contributes an amount $c_{i}$, her payoff is $v_{i}-c_{i}$ if $c_{1}+c_{2} \geq k$ (i.e. the project is completed) and $-c_{i}$ otherwise. The benefit $v_{i}$ is Player $i$ 's private information while the other maintains a prior that it is a random draw from a continuous distribution $F_{i}$ whose support is an interval $\left[\underline{v}_{i}, \bar{v}_{i}\right]$. Let $\mathcal{C}\left(F_{1}, F_{2}\right)$ denote the induced Bayesian game. A typical equilibrium of this game is a tuple $\left(C_{1}, C_{2}\right)$ where $C_{i}:\left[\underline{v}_{i}, \bar{v}_{i}\right] \longrightarrow \mathbb{R}$.

Two notable assumptions are implicit in the above description of the environment. First, the values of the players are independently distributed. This is an important restriction. Correlation between types warrants a separate analysis. Second, the benefit from not undertaking the project is normalized to zero. Therefore $\underline{v}_{i}$ is the minimum incremental benefit of Player $i$ from completing the project. We assume that this is non-negative. Also assume that $\bar{v}_{1}+\bar{v}_{2}>k$ to ensure that there are some valuations for which it is ex-post efficient to complete the project.

A more substantive restriction is our focus throughout to situations where no player has an incentive to complete the project on her own. Accordingly

$$
\bar{v}_{i}<k \quad \text { for } i=1,2
$$

is maintained throughout the paper.

(1) makes each player pivotal for the project. It is then immediate that neither player making a contribution regardless of her type is always an equilibrium. The question of interest is whether there are equilibria in which the project is completed with a positive probability. To develop a necessary and sufficient condition that answers this question, first define a "cost sharing" equilibrium as follows.

$\left(C_{1}, C_{2}\right)$ is a cost sharing equilibrium if for $i=1,2$,

$$
C_{i}\left(v_{i}\right)= \begin{cases}x_{i} & \text { if } v_{i} \geq \hat{v}_{i}, \\ 0 & \text { otherwise }\end{cases}
$$

for some non-negative $x_{i}$ such that $x_{1}+x_{2}=k$ and for some $\hat{v}_{i} \in\left[\underline{v}_{i}, \bar{v}_{i}\right)$.

In a cost sharing equilibrium a player contributes a constant $x_{i}$ if and only if her type is above a given threshold $\hat{v}_{i}$. With $\hat{v}_{i}<k$ and $x_{1}+x_{2}=k$, in a cost sharing equilibrium the project is completed with a positive probability. Lemma 1 below presents a simple necessary and sufficient condition for the existence of a cost sharing equilibrium. Proposition 1 then shows that the same condition applies to arbitrary equilibria. Define

$$
H\left(v_{1}, v_{2}\right)=\left(1-F_{2}\left(v_{2}\right)\right) v_{1}+\left(1-F_{1}\left(v_{1}\right)\right) v_{2} .
$$

Lemma 1. $\mathcal{C}\left(F_{1}, F_{2}\right)$ admits a cost sharing equilibrium if and only if there exists $\hat{v}_{i} \in\left[\underline{v}_{i}, \bar{v}_{i}\right)$ such that

$$
H\left(\hat{v_{1}}, \hat{v_{2}}\right) \geq k
$$


(2) is intuitive. Observe that $\left(1-F_{j}\left(\hat{v}_{j}\right)\right) \hat{v}_{i}$ is the expected benefit of the marginal type $\hat{v}_{i}$ should a cost sharing equilibrium exist. Therefore, $H\left(\hat{v}_{1}, \hat{v}_{2}\right)$ is merely the total expected benefit of the two marginal types in this equilibrium. The condition in the Lemma then says that a cost sharing equilibrium exists if and only if there is a pair of marginal types whose total expected benefit is at least as high as the cost of completing the project.

Proof of Lemma 1. Suppose a cost sharing equilibrium exists with $\left(v_{1}, v_{2}\right)$ being the tuple of marginal types and $\left(x_{1}, x_{2}\right)$ the respective shares of the cost. The expected payoff of type $v_{i}$ from contributing $x_{i}$ is $\left(1-F_{j}\left(v_{j}\right)\right) v_{i}-x_{i}$. Being the marginal type, she must weakly prefer contributing $x_{i}$ to not making a positive contribution, an action that results in a zero payoff. $H\left(v_{1}, v_{2}\right) \geq k$ is then merely the implication that the sum of these payoffs is non-negative.

Conversely, suppose (2) holds. Assume without loss of generality $3^{3}$ that the inequality is in fact binding for some $\left(v_{1}, v_{2}\right)$. Then define $x_{i}=\left(1-F_{j}\left(v_{j}\right)\right) v_{i}$ and conclude that only types $v_{i}$ and above making a positive contribution of $x_{i}$, for $i=1,2$, constitutes a cost sharing equilibrium.

The following notation is used at various places in the paper. Given an equilibrium $\left(C_{1}, C_{2}\right)$ in which the project is completed with a positive probability, let $\bar{c}_{i}$ and $\underline{c}_{i}$ respectively denote the supremum and the infimum of all the positive contributions among all types of Player $i$. Also let $\underline{w}_{i}$ denote the infimum of all types of Player $i$ that contribute a positive amount and $\bar{w}_{i}$ the infimum of all types of Player $i$ that choose $\bar{c}_{i}$. As we point out in the proof of Proposition 1, we must have $C_{i}\left(\bar{v}_{i}\right)=\bar{c}_{i}$ in any equilibrium. Therefore $\bar{w}_{i}$ is welldefined. Footnote 4 argues that $C_{i}(\cdot)$ is right continuous without loss of generality. Therefore $\underline{c}_{i}=C_{i}\left(\underline{w}_{i}\right)>0$.

Remark 1. We claim that the lowest positive contribution of one player and the highest contribution of the other must add up to the exact cost of the project. That is,

$$
\bar{c}_{i}+\underline{c}_{j}=k, \quad i \neq j \text { and } i, j=1,2 .
$$

(3) offers a testable hypothesis of equilibrium behavior. To see why it must be true, first note that since Player $j$ contributes at least $\underline{c}_{j}$, clearly $\bar{c}_{i} \leq k-\underline{c}_{j}$. Now suppose that the inequality is strict. By definition of $\underline{c}_{j}$, there must exist a type $v_{j}$ such that $C_{j}\left(v_{j}\right) \in\left(\underline{c}_{j}, k-\bar{c}_{i}\right)$. Then, by construction, $C_{j}\left(v_{j}\right)+C_{i}\left(v_{i}\right)<k$ for all $v_{i}$ which means that the marginal probability of the project's completion conditional on Player 2 contributing $C_{j}\left(v_{j}\right)$ is zero. Consequently, Player 2 is better off not to contribute at all than contributing $C_{j}\left(v_{j}\right)>0$. This contradiction establishes (3).

Proposition 1. The project is completed with a positive probability in some equilibrium of $\mathcal{C}\left(F_{1}, F_{2}\right)$ if and only if there exists $v_{i} \in\left[\underline{v}_{i}, \bar{v}_{i}\right)$ such that $H\left(v_{1}, v_{2}\right) \geq k$.

Proof of Proposition 1. If $H\left(v_{1}, v_{2}\right) \geq k$ for some $\left(v_{1}, v_{2}\right)$, by Lemma 1 a cost sharing equilibrium exists and establishes the proposition. To prove the converse, let $\left(C_{1}, C_{2}\right)$ be an equilibrium in which the project is completed with a positive probability. We will show that $H\left(\bar{w}_{1}, \underline{w}_{2}\right) \geq k$.

\footnotetext{
${ }^{3}$ If the inequality is strict, apply the intermediate value theorem to obtain a $\left(\hat{v}_{1}, \hat{v}_{2}\right)$ for which it is binding.
} 
For well known reasons of incentive compatibility, $C_{i}(\cdot)$ is necessarily non-decreasing. Consequently, there is no loss of generality in assuming that $C_{i}(\cdot)$ is also right continuous 4 The contribution $\bar{c}_{1}$ is thus made by player 1 of types in the interval $\left[\bar{w}_{1}, \bar{v}_{1}\right]$. By Remark 1 , with a contribution of $\bar{c}_{1}, \bar{c}_{1}+C_{2}\left(\tilde{v}_{2}\right) \geq k$ if and only if $\tilde{v}_{2} \geq \underline{w}_{2}$. Therefore, the equilibrium payoff of type $\bar{w}_{1}$ is

$$
\left(1-F_{2}\left(\underline{w}_{2}\right)\right) \bar{w}_{1}-\bar{c}_{1} .
$$

Since $\bar{c}_{1}$ is the highest contribution of Player 1 , it then follows that the probability that the project is completed when Player 2 contributes $\underline{c}_{2}$ is exactly $\left(1-F_{1}\left(\bar{w}_{1}\right)\right)$ and hence the equilibrium payoff of type $\underline{w}_{2}$ is

$$
\left(1-F_{1}\left(\bar{w}_{1}\right)\right) \underline{w}_{2}-\left(k-\bar{c}_{1}\right) .
$$

Noting that the expressions (4a) and (4b) must be non-negative and summing them yields $H\left(\bar{w}_{1}, \underline{w}_{2}\right) \geq k$.

Remark 2. With concave priors, it is possible to obtain a much simpler condition for which Lemma 1 obtains. Note that (2) really is of interest only when

$$
\underline{v}_{1}+\underline{v}_{2}<k .
$$

For, if $\underline{v}_{1}+\underline{v}_{2} \geq k$, it is always ex-post efficient to complete the project. When that is true, for Player 1 to contribute $\underline{v}_{1}$ and Player 2 to contribute $k-\underline{v}_{1}$ constitutes an equilibrium in which the project is completed with probability one, regardless of any further assumptions on the prior distributions. Interestingly, the converse also holds with concave priors.

Proposition 2. Suppose $F_{i}(\cdot)$ is concave for $i=1,2$ and (5) holds. Then, in the unique equilibrium of the contribution game, each player makes a zero contribution. The project is never completed.

Proof. Since $F_{i}(\cdot)$ is concave, $H(\cdot, \cdot)$ is convex in each of its arguments. Thus the maximum value of $H(\cdot, \cdot)$ is achieved at one of the four corners namely $\left(\underline{v}_{1}, \underline{v}_{2}\right),\left(\underline{v}_{1}, \bar{v}_{2}\right),\left(\bar{v}_{1}, \underline{v}_{2}\right)$ and $\left(\bar{v}_{1}, \bar{v}_{2}\right)$. The respective values of $H(\cdot, \cdot)$ at these points are $\underline{v}_{1}+\underline{v}_{2}, \bar{v}_{2}, \bar{v}_{1}$ and 0 . Thus $H\left(v_{1}, v_{2}\right)<k$ for all $\left(v_{1}, v_{2}\right)$. Apply Proposition 1 to complete the proof.

We end this section with one further property of equilibria of $\mathcal{C}\left(F_{1}, F_{2}\right)$.

Lemma 2. $\underline{w}_{1}+\underline{w}_{2}>k$ in any equilibrium of $\mathcal{C}\left(F_{1}, F_{2}\right)$ in which the project is completed with a positive probability.

Like (3), the inequality in the above lemma offers another testable hypothesis of equilibrium behavior. Equally important is the role it plays in establishing the results in Section 3

\footnotetext{
${ }^{4}$ Let $v_{i}$ be an arbitrary point of discontinuity of $C_{i}(\cdot)$ such that $C_{i}\left(v_{i}\right)<\lim _{v \downarrow v_{i}} C_{i}(v)=c^{*}$. Given that the types are continuously distributed, type $v_{i}$ must be indifferent between contributing $c^{*}$ and $C_{i}\left(v_{i}\right)$. Now let $\hat{C}_{i}(\cdot)$ be the function that differs from $C_{i}(\cdot)$ only at points of discontinuity such as $v_{i}$ and define $\hat{C}\left(v_{i}\right)=c^{*}$ described above. Since a monotonic function on a compact interval can have only countably many discontinuities, it follows that $\left(\hat{C}_{1}, \hat{C}_{2}\right)$ is also an equilibrium. Therefore $\left(\hat{C}_{1}, \hat{C}_{2}\right)$ induces the same set of equilibrium outcomes as $\left(C_{1}, C_{2}\right)$, except over a set with measure zero. Thus $\left(C_{1}, C_{2}\right)$ and $\left(\hat{C}_{1}, \hat{C}_{2}\right)$ induce the same outcomes except on a set of measure zero.
} 
Proof of Lemma 2. Let $\left(C_{1}, C_{2}\right)$ be an equilibrium in which the project is completed with a positive probability. Let $q_{i}=\left(1-F_{j}\left(\bar{w}_{j}\right)\right)$ and $p_{i}=\left(1-F_{j}\left(\underline{w}_{j}\right)\right)$. Without loss of generality let $q_{2} \geq q_{1}$. Since the project is completed with a positive probability, using (1) and Remark 1 . $q_{2}>0$. By individual rationality for type $\underline{w}_{i}$, we have $q_{i} \underline{w}_{i} \geq \underline{c}_{i}$ and hence $q_{2}\left(\underline{w}_{1}+\underline{w}_{2}\right) \geq$ $\underline{c}_{1}+\underline{c}_{2}$. Moreover, to satisfy the incentives of type $\bar{v}_{2}$ we have $p_{2} \bar{v}_{2}-\bar{c}_{2} \geq q_{2} \bar{v}_{2}-\underline{c}_{2}$. Substitute $\bar{c}_{2}=k-\underline{c}_{1}$ from Remark 1 to get $\underline{c}_{1}+\underline{c}_{2} \geq\left(q_{2}-p_{2}\right) \bar{v}_{2}+k$. Use (1) and $q_{2} \leq p_{2}$, then substitute $k$ for $\bar{v}_{2}$ on the RHS of this inequality to note that $\underline{c}_{1}+\underline{c}_{2} \geq q_{2}\left(1+\left(1-p_{2}\right) / q_{2}\right) k$.

Using the upper and lower bounds for $\underline{c}_{1}+\underline{c}_{2}$ in the above paragraph, we obtain that $\underline{w}_{1}+\underline{w}_{2} \geq\left(1+\left(1-p_{2}\right) / q_{2}\right) k>k$.

\section{CheAP TALK}

We now turn to implications of a prior stage of non-binding communication to $\mathcal{C}\left(F_{1}, F_{2}\right)$. Each player is assigned a binary message space $M=\{y, n\}$ where $y$ stands for the announcement "Yes, I will make a contribution" and $n$ stands for "No, I will not contribute" to the project's cost. Having observed their types, players simultaneously and publicly announce $y$ or $n$. This is followed by a play of the contribution mechanism of the previous section. Let $\mathcal{C}^{*}\left(F_{1}, F_{2}\right)$ denote the induced multistage game with observed actions. By an equilibrium of $\mathcal{C}^{*}\left(F_{1}, F_{2}\right)$, we mean a Perfect Bayesian Equilibrium $(\mathrm{PBE})^{5}$.

It is worth reiterating that our interest here is not in the entire gamut of outcomes that can be achieved through an equilibrium of some cheap talk extension of $\mathcal{C}\left(F_{1}, F_{2}\right)$. See Section 4.2 in this regard. Rather, the idea here is to demonstrate that even limited communication of two messages can lead to superior efficiency despite the fact that $\mathcal{C}\left(F_{1}, F_{2}\right)$ involves a continuum of types and actions. What is more, such superior efficiency can be achieved in equilibria of $\mathcal{C}^{*}\left(F_{1}, F_{2}\right)$ that have the following simple structure.

Definition 1 (Simple Strategy Profile of $\left.\mathcal{C}^{*}\left(F_{1}, F_{2}\right)\right)$. A strategy profile in $\mathcal{C}^{*}\left(F_{1}, F_{2}\right)$ is said to be simple if there exists $x$ such that

1. At the communication stage

- Player 1 announces $y$ only if her type is at least $x$

- Player 2 announces $y$ only if her type is at least $k-x$

2. At the contribution stage, neither player makes a positive contribution unless both have previously announced $y$. If both have said $y$ then

- Player 1 contributes $x$ if her type is at least $x$.

- Player 2 contributes $k-x$ if her type is at least $k-x$

Let $S(x)$ denote a simple strategy profile in which Player 1 contributes $x$. We shall say that an equilibrium of $\mathcal{C}^{*}\left(F_{1}, F_{2}\right)$ is a simple equilibrium if it involves a simple strategy profile. We

\footnotetext{
${ }^{5}$ The reader may consult any standard text, such as Fudenberg and Tirole (1991) for details of a multistage game and its' PBE.
} 
will use the same notation $\mathcal{S}(x)$ to denote any simple equilibrium in which the corresponding simple strategy is played. The following Lemma settles the question of existence of simple equilibria.

Lemma 3. $\mathcal{S}(x)$ is a simple equilibrium if and only if $x \in\left[k-\bar{v}_{2}, \bar{v}_{1}\right]$.

Proof. See Appendix.

Remark 3. As discussed earlier, when (5) is violated, the project can be completed with probability one even without cheap talk. To keep the role of cheap talk interesting, we assume (5) in what follows.

\subsection{Cheap Talk and probability of completion}

To see that cheap talk can sometimes help, it is enough to consider $\mathcal{C}\left(F_{1}, F_{2}\right)$ when the priors are concave. Absent communication, by Proposition 2, the unique equilibrium is for neither player to contribute. In contrast, in any simple equilibrium $\mathcal{S}(x)$, the project is completed whenever $v_{1} \geq x$ and $v_{2} \geq k-x$, and hence with the positive probability $\left(1-F_{1}(x)\right)(1-$ $\left.F_{2}(k-x)\right)$ for any $x \in\left(k-\bar{v}_{2}, \bar{v}_{1}\right)$. Interestingly, the next result shows that such an increase in probability of completion extends to arbitrary priors. Let

$$
x_{p} \in \arg \max _{x \in\left[k-\bar{v}_{2}, \bar{v}_{1}\right]}\left(1-F_{1}(x)\right)\left(1-F_{2}(k-x)\right) .
$$

$\mathcal{S}\left(x_{p}\right)$ is a simple equilibrium under which the project is completed with the highest probability compared to all other simple equilibria of the cheap talk game $\mathcal{C}^{*}\left(F_{1}, F_{2}\right)$.

Proposition 3. The project is completed with a greater probability in the simple equilibrium $\mathcal{S}\left(x_{p}\right)$ of the cheap talk game than in any equilibrium of the contribution game without cheap talk.

Proof of the above proposition relies on Lemma 2, Due to (1), this Lemma implies that the project is not completed unless Player $i$ 's type is at least $\underline{w}_{i}$, for both $i=1,2$. In contrast, in the simple equilibrium, $\mathcal{S}\left(\underline{w}_{2}\right)$, the project is completed for additional realizations $\mathrm{v}=\left(v_{1}, v_{2}\right)$ when $k-\underline{w}_{2} \leq v_{1} \leq \underline{w}_{1}$ and $v_{2} \geq \underline{w}_{2}$. This essentially constitutes the proof of Proposition 3 .

Proof of Proposition 3. The project is completed with probability $\left(1-F_{1}\left(x_{p}\right)\right)\left(1-F_{2}(k-\right.$ $\left.x_{p}\right)$ ) in the equilibrium $\mathcal{S}\left(x_{p}\right)$. In an equilibrium $\left(C_{1}, C_{2}\right)$ of the game without cheap talk, the probability that the project is completed is no more than $\left(1-F_{1}\left(\underline{w}_{1}\right)\right)\left(1-F_{2}\left(\underline{w}_{2}\right)\right)$. By Lemma 2, we have $\underline{w}_{2}>k-\underline{w}_{1}$ and thus the probability of completion is less than $\left(1-F_{1}\left(\underline{w}_{1}\right)\right)\left(1-F_{2}\left(k-\underline{w}_{1}\right)\right)$ which in turn, by definition of $x_{p}$, is no greater than probability of completion in the equilibrium $\mathcal{S}\left(x_{p}\right)$.

Remark 4. Recall that a probability distribution $F$ is said to be regular if $(1-F(t)) / f(t)$ is decreasing, where $f$ is the density of $F$. If types are i.i.d according to a regular $F$, it may be verified that $x_{p}=k / 2$. In other words, for regular distributions, the simple strategy in which the costs are shared equally also maximizes the probability of completion of the project. 


\subsection{Cheap Talk and Efficiency}

We now evaluate the superiority of simple equilibria in terms of their payoffs rather than the probability of completion. The various notions of efficiency that we study are standard in the literature but are listed below for completeness.

Given an equilibrium $\mathcal{E}$ (of either $\mathcal{C}\left(F_{1}, F_{2}\right)$ or $\mathcal{C}^{*}\left(F_{1}, F_{2}\right)$ ) let $U_{i}^{\mathcal{E}}\left(v_{i}\right)$ denote the interim payoff of Player $i$ of type $v_{i}$ with $U_{i}^{\mathcal{E}}=E\left[U_{i}^{\mathcal{E}}\left(v_{i}\right)\right]$ denoting her ex-ante expected payoff.

Definition 2 (Interim Pareto Dominance). An equilibrium $\mathcal{E}$ is said to interim Pareto dominate another equilibrium $\hat{\mathcal{E}}$ if $U_{i}^{\hat{\mathcal{E}}}\left(v_{i}\right) \geq U_{i}^{\mathcal{E}}\left(v_{i}\right)$ for $i=1,2$ and for almost all $v_{i} \in\left[\underline{v}_{i}, \bar{v}_{i}\right]$ with the inequality being strict for a generic subset of types of each player.

Definition 3 (Ex-ante Pareto Dominance). An equilibrium $\mathcal{E}$ is said to ex-ante Pareto (strictly) dominate another equilibrium $\hat{\mathcal{E}}$ if $U_{i}^{\hat{\mathcal{E}}}>U_{i}^{\mathcal{E}}$ for $i=1,2$.

Observe that when comparing one equilibrium with another, we require that both players are made strictly better off. It is easy to check that if an equilibrium $\mathcal{E}$ is interim Pareto dominated by another equilibrium $\hat{\mathcal{E}}$, then the latter also ex-ante Pareto dominates $\mathcal{E}$. The converse of course is not true in general.

Proposition 4. For every equilibrium of the contribution game, there exists a simple equilibrium of the cheap talk game that dominates it in the Interim-Pareto (and hence in the Ex-ante Pareto) sense.

Proof. See Appendix.

Again, Lemma 2 is the key ingredient to the proof Proposition 4. Intuitively, as in the case of Proposition 3 the additional ex-post efficient states in which the project is completed due to communication enhance efficiency.

It is important to note that the choice of the dominating simple strategy in the above proposition will usually depend on the particular equilibrium of $\mathcal{C}\left(F_{1}, F_{2}\right)$ that one is seeking to dominate. In general, it is not possible for any one simple equilibrium to uniformly dominate all the equilibria of the contribution game 6 Uniform dominance is however possible if an aggregate welfare based on the ex-ante utilities of the two players is used to rank equilibria.

Let $\phi: \mathbb{R} \times \mathbb{R} \longrightarrow \mathbb{R}$ be a social welfare function and $\phi\left(U_{1}^{\mathcal{E}}, U_{2}^{\mathcal{E}}\right)$ be the aggregate welfare in an equilibrium $\mathcal{E}$ of $\mathcal{C}\left(F_{1}, F_{2}\right)$ or $\mathcal{C}^{*}\left(F_{1}, F_{2}\right)$.

Proposition 5. Suppose that welfare is measured according to a continuous function $\phi(\cdot, \cdot)$ that is strictly increasing in both its arguments. Then, there exists $x_{\phi} \in\left[k-\bar{v}_{2}, \bar{v}_{1}\right]$, such that welfare in the simple equilibrium $\mathcal{S}\left(x_{\phi}\right)$ is greater than the welfare in any equilibrium of the contribution game.

Proof of Proposition 5. Let $\mathcal{E}$ be an equilibrium of $\mathcal{C}\left(F_{1}, F_{2}\right)$ and apply Proposition 4 to choose an equilibrium $\mathcal{S}(x)$ such that ex-ante Pareto dominates $\mathcal{E}$. Since $\phi(\cdot, \cdot)$ is increasing in both its arguments, $\phi\left(U_{1}^{\mathcal{S}(x)}, U_{2}^{\mathcal{S}(x)}\right)>\phi\left(U_{1}^{\mathcal{E}}, U_{2}^{\mathcal{E}}\right)$. Choose $x_{\phi} \in \arg \max _{x \in\left[k-\bar{v}_{2}, \bar{v}_{1}\right]} \phi\left(U_{1}^{\mathcal{S}(x)}, U_{2}^{\mathcal{S}(x)}\right)$ to complete the proof.

\footnotetext{
${ }^{6}$ With concave priors, however, such a uniform dominance is possible since by Proposition 2 zero contribution is the unique equilibrium outcome of the contribution game.
} 
A much stronger characterization obtains if one assumes that priors are regular (See Remark 4) and that the welfare criterion is utilitarian. First define

$$
\psi_{i}(x)=\frac{1}{f_{i}(x)} \int_{x}^{\bar{v}_{i}}\left(1-F_{i}(t)\right) d t \quad i=1,2 .
$$

Proposition 6. Suppose welfare is measured according to the function $\phi\left(u_{1}, u_{2}\right)=u_{1}+u_{2}$. Also, assume that $F_{i}$ is regular for $i=1,2 . x_{\phi}$ in Proposition 5 is the (unique) solution to the equation $\psi_{1}(x)=\psi_{2}(k-x)$. Moreover, if $F_{1}=F_{2}$ then $x_{\phi}=k / 2$.

Proof. See Appendix.

\section{Discussion}

In this section, we briefly discuss various aspects of our work.

\subsection{Efficiency of Simple Equilibria}

In Section 3.1 and Section 3.2 , we have shown that simple equilibria have attractive properties when compared to the equilibrium outcomes without communication. Can simple equilibria in turn be dominated by other equilibria of $\mathcal{C}^{*}\left(F_{1}, F_{2}\right)$ ? We do not have an answer to this question for general distribution functions. The next result is the answer for a class of concave priors.

Let $x_{i}^{*} \equiv \arg \max _{x \in\left[k-\bar{v}_{2}, \bar{v}_{1}\right]} U_{i}^{\mathcal{S}(x)}$. That is, among all simple equilibria, Player $i$ 's ex-ante payoff is the highest in $\mathcal{S}\left(x_{i}^{*}\right)$.

Proposition 7. Assume that $F_{1}, F_{2}$ are both concave and $\underline{v}_{i}+\bar{v}_{j} \leq k, i \neq j$. An equilibrium of $\mathcal{C}^{*}\left(F_{1}, F_{2}\right)$ is interim efficient if and only if it is a simple equilibrium.

Moreover, if for $i=1,2, F_{i}$ is regular, then $x_{1}^{*}<x_{2}^{*}$ and a simple equilibrium $\mathcal{S}(x)$ is ex-ante efficient if and only if $x \in\left[x_{1}^{*}, x_{2}^{*}\right]$.

Proof. See Appendix.

\subsection{Inefficiency of Simple Equilibria}

Our analysis thus far has focused on how even limited communication in the form of simple equilibria can enhance efficiency. In this section, we shall briefly discuss efficient outcomes when one allows arbitrary communication schemes. In particular, our analysis will also highlight the (in)efficiency of the simple equilibria themselves in this more elaborate class. Throughout, we shall restrict our analysis to the case where $F_{1}=F_{2}=F$ is the uniform distribution on $[\underline{v}, \bar{v}]$.

Recall from the revelation principle, that an arbitrary procedure for deciding how to undertake the project can be described by a direct mechanism, i.e. a tuple $\mu:=\left\{p(\cdot), x_{1}(\cdot), x_{2}(\cdot)\right\}$ where $p(\mathrm{v})$ is the probability that the project is completed and $x_{i}(\mathrm{v})$ is the expected payment of Player $i$ following the reports $\mathrm{v}=\left(v_{1}, v_{2}\right)$. Let $\mathcal{M}$ denote the set of all $\mu$ that satisfy 
incentive compatibility, interim individual rationality and ex-ante budget balance 7 . Let $\mathcal{P}$ denote the subset of $\mathcal{M}$ that are ex-ante efficient.

Let $\mathcal{C}^{* *}$ denote the cheap talk extension of $\mathcal{C}(F, F)$ where players first publicly report a type and, having observed the reports, play the contribution game. The next result shows that every $\mu \in \mathcal{P}$ can be achieved as a fully revealing equilibrium of $\mathcal{C}^{* *}$.

Given $\alpha<\bar{v}$, let $g(\alpha)=3 k / 2-\bar{v}-\alpha$ and $p^{\alpha}(\cdot)$ be the rule according to which the project is completed (with probability one) only when $\left(v_{1}, v_{2}\right)$ lies above the line joining $(\alpha, \bar{v})$ and $(\bar{v}, g(\alpha))$. That is, $p^{\alpha}(\mathrm{v})=1$ if $v_{2} \geq m_{\alpha} v_{1}+c_{\alpha}$ and $p^{\alpha}(\mathrm{v})=0$ otherwise, where $m_{\alpha}=-(2 \bar{v}+\alpha-3 / 2 k) /(\bar{v}-\alpha)$ and $c_{\alpha}=\bar{v}-m_{\alpha} \alpha$.

Let $a_{1}=\left(2+m_{a}\right) / 3, a_{2}=-\left(1+2 m_{\alpha}\right) / 3 m_{\alpha}$ and $a_{3}=\left(\alpha-a_{2}\left(\bar{v}+c_{a}\right)\right) / 2$

Proposition 8. Suppose $\underline{v}+\bar{v}<k$ and $F_{1}=F_{2}=F$ is the uniform distribution on $[\underline{v}, \bar{v}]$.

1. $\mu:=\left\{p(\cdot), x_{1}(\cdot), x_{2}(\cdot)\right\} \in \mathcal{P}$ if and only if $p(\cdot)=p^{\alpha}(\cdot)$ for some $\alpha \in[k-\bar{v}, k / 2]$.

2. For each $\alpha \in[k-\bar{v}, k / 2], p^{\alpha}(\cdot)$ is achieved in the following FCE of $\mathcal{C}^{* *}$ : Players truthfully report their types at the communication stage. At the contribution stage, Player 1 contributes $x_{1}(v)=a_{1} v_{1}+a_{2} v_{2}+a_{3}$ and and Player 2 contributes $x_{2}(v)=k-x_{1}(v)$ if the reports $v=\left(v_{1}, v_{2}\right)$ satisfy $v_{2} \geq m_{a} v_{1}+c_{a}$ and their types are at least as high. In all other cases, neither player makes a contribution.

Proof. See Appendix.

Several remarks are in order. Denote by $C^{*}\left(M_{1}, M_{2}\right)$ a cheap talk extension of $\mathcal{C}(F, F)$ with arbitrary message spaces $M_{1}$ and $M_{2}$. An equilibrium of this game is a full-communication equilibrium (FCE) if the types are fully revealed at the communication stage. Let $\mathcal{M}^{*}$ denote the set of all $\mu$ that can be achieved as the FCE of $\mathcal{C}^{*}\left(M_{1}, M_{2}\right)$ for some $M_{1}, M_{2}$. Note that $\mathcal{M}^{*}$ is a subset 8 of $\mathcal{M}$. The above proposition is therefore a complete characterization of all the efficient outcomes that can be obtained by adding an arbitrary communication stage to $\mathcal{C}(F, F)$. It is also noteworthy that complicated message spaces are not necessary - players need only communicate their types - to achieve efficiency.

Next, no simple strategy is ex-ante efficient. To see this, recall that under $p^{\alpha}(\cdot)$ the project is completed only when $\left(v_{1}, v_{2}\right)$ lies on or above the line joining $(\alpha, \bar{v})$ and $(\bar{v}, g(\alpha))$. As $\alpha$ varies over $[k-\bar{v}, k / 2]$, the lower envelope of these lines describes a curve $9^{9} \mathbb{C}$ below which the project is not completed in any ex-ante efficient FCE. When $\alpha=k-\bar{v}, \mathbb{C}$ passes through $(k-\bar{v}, \bar{v})$ and has a slope $m_{\alpha}=-1 / 2$. The tangent line $\ell_{1}$ through this point is shown in the figure below. As $\alpha$ increases to $\alpha=k / 2$, it passes through $(\bar{v}, k-\bar{v})$ with its slope decreasing continuously to $m_{\alpha}=-2$. The tangent line at this point is shown as $\ell_{2}$. Therefore the bold line depicts $\mathbb{C}$ in the figure below. Observe that $\mathbb{C}$ remains bounded away from the line $v_{1}+v_{2}=k$ except at $(k-\bar{v}, \bar{v})$ and $(\bar{v}, k-\bar{v})$. On the other hand, it is clear

\footnotetext{
${ }^{7}$ That is expected payments cover the expected cost of the project $E\left[x_{1}(\mathrm{v})+x_{2}(\mathrm{v})-p(\mathrm{v}) k\right] \geq 0$

${ }^{8}$ In any FCE, the types are fully revealed at the communication stage. Since not contributing is always a possible deviation and the project is only completed if adequate contributions are made ex-post IR and ex-post budget balance must hold as follows. That is, $p(\mathrm{v}) v_{i}-x_{i}(\mathrm{v}) \geq 0$ and $x_{1}(\mathrm{v})+x_{2}(\mathrm{v}) \geq q(\mathrm{v}) k$. Consequently interim IR and ex-ante budget balance are satisfied.

${ }^{9}$ Formally, for each $v_{1} \geq k-\bar{v}$, let $\hat{g}\left(v_{1}\right)=\min _{\alpha \in[k-\bar{v}, k / 2]} m_{\alpha} v_{1}+c_{\alpha}$. Then $\mathbb{C}=\{(\alpha, \hat{g}(\alpha)): \alpha \in[k-\bar{v}, \bar{v}]\}$.
} 
that in every simple equilibrium, the project is undertaken in some part of the shaded region that lies between the line $v_{1}+v_{2}=k$ and $\mathbb{C}$. Therefore, no simple equilibrium is ex-ante efficient.

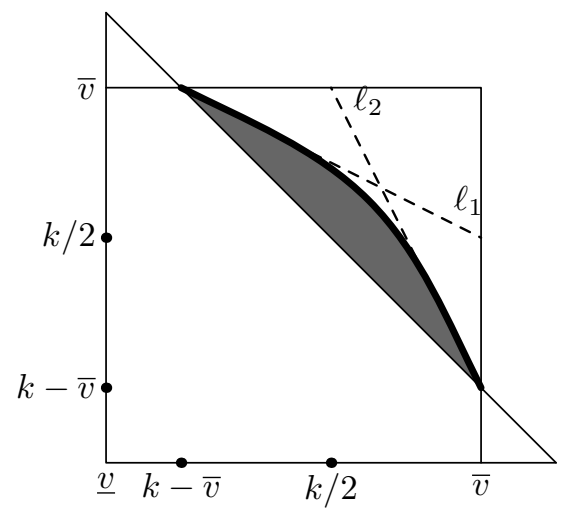

Figure 1: Project is not completed in the shaded region in any ex-ante efficient allocation. In every simple equilibrium, the project is completed in some part of the shaded region.

Finally, a comment on the assumption of uniform priors. In order to prove Proposition 8 , we rely on Williams (1987). It turns out that the programming problem for characterizing $\mathcal{P}$ is isomorphic to the one studied by Williams (1987) for characterizing incentive efficient mechanisms for bilateral trade. Therefore, an analogue of Part 1 of the Proposition can in fact be stated for all regular priors. However, Part 2 requires an explicit construction of a FCE of some $\mathcal{C}^{*}\left(M_{1}, M_{2}\right)$. This appears substantially intricate unless priors are uniform. We leave this as an avenue for future research. The assumption that $\underline{v}+\bar{v} \leq k$ (for uniform priors) is however only a simplifying assumption to avoid a cumbersome analysis of several cases. (The inefficiency of simple strategies discussed above remains valid.)

\subsection{One sided communication}

$\mathcal{C}^{*}\left(F_{1}, F_{2}\right)$ was presented in Section 3 largely with the intent of showing how a "simple" communication scheme and "simple" strategies of that scheme can dominate all equilibria of the original contribution game. It is only reasonable to ask if there even simpler mechanisms that can lead to a similar result. For example, is there a cheap talk extension in which only one player chooses from a binary message space and has equilibria with similar efficiency properties. The following argument shows that in general, communication by both players is necessary.

Assume, without loss of generality, that it is Player 1 who first publicly reports $y$ or $n$ after which the contribution mechanism is played. Assume that the priors are symmetric and concave on $[0,1]$ and that $k>1$. For an arbitrary $v_{1}^{*} \in[0,1]$, consider an equilibrium strategy of Player 1 in which she announces $y$ if and only if her valuation is at least $v^{*}$. Then following the cheap talk phase, when the contribution game is played at the node $y$, the lowest possible valuation of Player 1 according to the revised prior is $v_{1}^{*}$, while that of Player 2 continues to be 0 . Since the sum of these valuations $v_{1}^{*}<k$, it follows from Proposition 2 that neither player will make a positive contribution. Likewise at the node following the announcement of $n$ by Player 1 . Therefore, in the original game and this cheap talk extension with one sided 
communication, the project is never completed.

\subsection{More than two players}

Many (though not all) of our results have an analogue in the case of $n$ players provided one assumes that no subset of $n-1$ players have an incentive to complete the project. While this is the condition (1) in the case of $n=2$, it is substantially more stringent when $n>2$. A more complete study of the situation involving $n$ players can be a subject of future research.

\section{APPENDIX}

Remark 5. Recall that given (1), for neither player to contribute is always an equilibrium regardless of any further assumptions on the prior distributions. We shall refer to this as 0-equilibrium.

Proof of Lemma 3. Strategies at the communication stage result in one of four histories: $(n, n),(n, y),(y, n)$ and $(y, y)$, the two coordinates being the respective announcements of the two players. Following any history other than $(y, y)$, the restriction of $\mathcal{S}(x)$ to the continuation game requires that neither player make a positive contribution. To support this outcome, it is irrelevant as to how one assigns the posterior beliefs at these nodes.

Since $\bar{v}_{1}+\bar{v}_{2}>k$, it is immediate that $x<\bar{v}_{1}$ and $k-x<\bar{v}_{2}$. Therefore, for every $x \in\left[k-\bar{v}_{2}, \bar{v}_{1}\right]$, the node $(y, y)$ occurs with a positive probability under the strategy profile $\mathcal{S}(x)$. The continuation game at this node is $\mathcal{C}\left(\tilde{F}_{1}, \tilde{F}_{2}\right)$ where $\tilde{F}_{i}$ is the truncation of $F_{i}$ to $\left[x_{i}, \bar{v}_{i}\right], x_{1}=x$ and $x_{2}=k-x$. Take the posterior beliefs that is consistent with Bayes rule at this node. Given her beliefs, and the restriction of $\mathcal{S}(x)$ to this game, Player $i$ knows that Player $j$ will contribute $x_{j}$ for sure. Therefore it is clearly a best response for her to contribute $x_{i}$ as long as her valuation exceeds $x_{i}$ which is the specification in $\mathcal{S}(x)$.

It remains to check that there are no incentives to deviate at the initial announcement stage. Consider player 1 . We will show that type $x$ of Player 1 is indifferent between sending the messages $y$ and $n$. Now sending the message $n$ leads to a payoff of zero as the project is not undertaken nor any contributions are made by either player. Upon sending the message $y$, if called upon to contribute a positive amount, her contribution is $x$, which again yields a zero payoff. Clearly, then all types of player 1 exceeding $x$ will strictly prefer to to send the message $y$ while there is no benefit of a type less than $x$ since in the continuation game, it will need too contribute at least $x$ for the completion of the project. Thus player 1's announcement choice in the cheap talk game is optimal. A similar argument establishes that player $2^{\prime} s$ choice of $y$ if her type exceeds $k-x$ and $n$ otherwise is optimal.

The requirement that $x \leq \bar{v}_{1}$ and $k-x \leq \bar{v}_{2}$ for $\mathcal{S}(x)$ to be an equilibrium is immediate as otherwise at least one of the players will be expected to contribute an amount greater than the valuation of the highest type.

Given an equilibrium $\mathcal{E}$ of either $\mathcal{C}\left(F_{1}, F_{2}\right)$ or $\mathcal{C}^{*}\left(F_{1}, F_{2}\right)$, let $q_{i}^{\mathcal{E}}(v)$ be the marginal probability that the project is completed given that Player $i$ is of type $v$. The following lemma 
which is required for the proof of the above proposition is essentially due to Myerson (1981) and is stated without proof.

Lemma 4. Let $\mathcal{E}$ be an equilibrium of either $\mathcal{C}\left(F_{1}, F_{2}\right), \mathcal{C}^{*}\left(F_{1}, F_{2}\right)$. Given any $\hat{v}_{i} \in\left[\underline{v}_{i}, \bar{v}_{i}\right]$, the equilibrium payoff of type $v_{i} \geq \hat{v}_{i}$ is given by

$$
U_{i}\left(v_{i}\right)=U_{i}\left(\hat{v}_{i}\right)+\int_{\hat{v}_{i}}^{v_{i}} q_{i}^{\mathcal{E}}(t) d t, \text { for all } v_{i} \in\left[\hat{v}_{i}, \bar{v}_{i}\right] .
$$

Proof. Omitted.

Proof of Proposition 4. Consider an equilibrium $\mathcal{E}$ of $\mathcal{C}\left(F_{1}, F_{2}\right)$. As the project cannot be completed with probability one, at least one player with the lowest possible valuation does not make a contribution. Assume then, without loss of generality, that $\underline{w}_{1}>\underline{v}_{1}$ so that $U_{1}^{\mathcal{E}}\left(\underline{w}_{1}\right)=0$. Noting that the marginal probability of completion of the project given any equilibrium contribution of Player 1 does not exceed $\left(1-F_{2}\left(\underline{w}_{2}\right)\right)$, by applying Lemma 4 the interim payoffs of Player 1 must satisfy the following:

$$
U_{1}^{\mathcal{E}}\left(v_{1}\right) \begin{cases}=0 & \text { if } v_{1} \in\left[\underline{v}_{1}, \underline{w}_{1}\right) \\ \leq\left(v_{1}-\underline{w}_{1}\right)\left(1-F_{2}\left(\underline{w}_{2}\right)\right) & \text { if } v_{1} \in\left[\underline{w}_{1}, \bar{v}_{1}\right]\end{cases}
$$

Turning to Player 2, the payoff of type $\underline{w}_{2}$ of Player 2 is $U_{2}^{\mathcal{E}}\left(\underline{w}_{2}\right)=\left(1-F_{1}\left(\bar{w}_{2}\right)\right) \underline{w}_{2}-\underline{c}_{2}$ which is zero if $\underline{w}_{2}>\underline{v}_{2}$ but can be positive if $\underline{w}_{2}=\underline{v}_{2}$. Again, by Lemma 4 , the interim payoffs of Player 2 must satisfy the following:



From Lemma 2, we know that $\underline{w}_{1}+\underline{w}_{2}>k$. Therefore, $k-\bar{v}_{2}<\underline{w}_{1}<\bar{v}_{1}$ and hence by Lemma $3, \mathcal{S}\left(\underline{w}_{1}\right)$ is a simple equilibrium. Let $a=\max \left\{k-\underline{w}_{1}, \underline{v}_{2}\right\}$. The interim payoffs in this equilibrium can be computed directly and are as follows:

$$
\begin{aligned}
& U_{1}^{\mathcal{S}\left(\underline{w}_{1}\right)}\left(v_{1}\right)= \begin{cases}0 & \text { if } v_{1} \in\left[\underline{v}_{1}, \underline{w}_{1}\right) \\
\left(v_{1}-\underline{w}_{1}\right)\left(1-F_{2}\left(k-\underline{w}_{1}\right)\right) & \text { if } v_{1} \in\left[\underline{w}_{1}, \bar{v}_{1}\right]\end{cases} \\
& U_{2}^{\mathcal{S}\left(\underline{w}_{1}\right)}\left(v_{2}\right)= \begin{cases}0 & \text { if } v_{2} \in\left[\underline{v}_{2}, a\right), \\
\left(v_{2}-\left(k-\underline{w}_{1}\right)\right)\left(1-F_{1}\left(\underline{w}_{1}\right)\right) & \text { if } v_{2} \in\left[a, \bar{v}_{2}\right]\end{cases}
\end{aligned}
$$

Since $\underline{w}_{1}+\underline{w}_{2}>k$, comparing (8a) and $(9 \mathrm{a})$ it is immediate that all types of Player 1 in the region $\left[\underline{w}_{1}, \bar{v}_{1}\right]$ are better off under $\mathcal{S}\left(\underline{w}_{1}\right)$ relative to $\mathcal{E}$ while its remaining types are no worse off.

Now comparing $(8 \mathrm{~b})$ and $(9 \mathrm{~b})$, the types of Player 2 in the region $\left[\underline{v}_{2}, a\right)$ receive a zero payoff in both equilibria while those types between $\left[a, \underline{w}_{2}\right)$ are clearly better off under $\mathcal{S}\left(\underline{w}_{1}\right)$ relative to $\mathcal{E}$. We now claim that the types in $\left[\underline{w}_{2}, \bar{v}_{2}\right]$ are also better off under $\mathcal{S}\left(\underline{w}_{1}\right)$. Let 
$\hat{v}_{2}$ satisfy $\left(1-F_{1}\left(\bar{w}_{1}\right)\right) \hat{v}_{2}=\underline{c}_{2}$. Clearly $\hat{v}_{2} \leq \underline{w}_{2}$. Consider now $v_{2} \in\left[\hat{v}_{2}, \bar{v}_{2}\right]$ the difference $U^{\mathcal{S}\left(\underline{w}_{1}\right)}\left(v_{2}\right)-U^{\mathcal{E}}\left(v_{2}\right)$ is at least

$$
\begin{aligned}
& \left(1-F_{1}\left(\underline{w}_{1}\right)\right)\left(v_{2}-\left(k-\underline{w}_{1}\right)\right) \\
& -\left[\left(1-F_{1}\left(\bar{w}_{1}\right)\right) \underline{w}_{2}-\underline{c}_{2}\right]-\left(v_{2}-\hat{v}_{2}\right)\left(1-F_{1}\left(\underline{w}_{1}\right)\right) \\
& \quad=\left(1-F_{1}\left(\underline{w}_{1}\right)\right)\left(\underline{w}_{1}+\underline{w}_{2}-k\right)-\left[\left(1-F_{1}\left(\bar{w}_{1}\right)\right) \hat{v}_{2}-\underline{c}_{2}\right] \\
& \quad=\left(1-F_{1}\left(\underline{w}_{1}\right)\right)\left(\underline{w}_{1}+\underline{w}_{2}-k\right) .
\end{aligned}
$$

From Lemma 3 , we know that $\underline{w}_{1}+\underline{w}_{2}>k$ and thus for all $v_{2} \in\left[\hat{v}_{2}, \bar{v}_{2}\right], U^{\mathcal{S}\left(\underline{w}_{1}\right)}\left(v_{2}\right)-U^{\mathcal{E}}\left(v_{2}\right) \geq$ 0 .

Thus, we have shown that for $i=1,2$, all the types of Player $i$ in the region $\left[\underline{w}_{i}, \bar{v}_{i}\right]$ obtain a higher payoff under $\mathcal{S}\left(\underline{w}_{1}\right)$ relative to $\mathcal{E}$. Since $\underline{w}_{i}<\bar{v}_{i}, \mathcal{S}\left(\underline{w}_{1}\right)$ interim and ex-ante dominates $\mathcal{E}$.

The following lemma identifies certain properties of regular distributions which are used in the proofs of Proposition 6 and Proposition 7.

Lemma 5. Let $G(t)=(1-F(t))$ where $F$ is a regular distribution on $[\underline{v}, \bar{v}]$. Then the following hold:

$$
\int_{x}^{\bar{v}} G(t) d t<\frac{G(x)^{2}}{f(x)} \quad \text { and }-1-\frac{f^{\prime}(x) G(x)}{f(x)^{2}}<0
$$

Moreover the function $\psi(x)=\frac{1}{f(t)} \int_{x}^{\bar{v}} G(t) d t$ in decreasing in $x$.

Proof. By assumption, $G(\cdot) / f(\cdot)$ is decreasing. The second inequality in 10 must hold as its LHS is merely the derivative of $G(x) / f(x)$. The first inequality is an be obtained by first multiplying and dividing the integrand on its LHS by $f(t)$ and using the fact that $G(t) / f(t)$ is decreasing. Finally, differentiate $\psi(\cdot)$ to get

$$
\begin{aligned}
\psi^{\prime}(x) & =-\frac{G(x)}{f(x)}-\frac{f^{\prime}(x)}{f(x)^{2}} \int_{x}^{\bar{v}} G(t) d t \\
& <\frac{G(x)}{f(x)}\left[-1-\frac{f^{\prime}(x) G(x)}{f(x)^{2}}\right]<0
\end{aligned}
$$

Therefore, $\psi(\cdot)$ is decreasing.

Proof of Proposition 6. The interim payoffs in the equilibrium $\mathcal{S}(x)$ are as follows:

$$
\begin{aligned}
& U_{1}^{\mathcal{S}(x)}\left(v_{1}\right)= \begin{cases}0 & \text { if } v_{1} \leq x \\
\left(1-F_{2}(k-x)\right)\left[v_{1}-x\right] & \text { if } v_{1} \geq x\end{cases} \\
& U_{2}^{\mathcal{S}(x)}\left(v_{2}\right)= \begin{cases}0 & \text { if } v_{2} \leq k-x \\
\left(1-F_{1}(x)\right)\left[v_{2}+x-k\right] & \text { if } v_{2} \geq k-x\end{cases}
\end{aligned}
$$


Use the above expressions and take the expectation of the interim payoffs $U_{i}^{\mathcal{S}(x)}(\cdot)$ and then apply integration by parts to obtain the following expressions for the ex-ante payoff $W_{i}(x) \equiv$ $E\left[U_{i}^{\mathcal{S}(x)}\left(v_{i}\right)\right]$

$$
W_{1}(x)=G_{2}(k-x) \int_{x}^{\bar{v}_{1}} G_{1}(t) d t \quad W_{2}(x)=G_{1}(x) \int_{k-x}^{\bar{v}_{1}} G_{2}(t) d t
$$

in the equilibria $\mathcal{S}(x)$, where $G_{i}(x)=\left(1-F_{i}(x)\right)$, for $i=1,2$. Let $B(x) \equiv \phi\left(U_{1}^{x}, U_{2}^{x}\right)=$ $W_{1}(x)+W_{2}(x)$. Differentiating $B(\cdot)$ and simplifying yields

$$
B^{\prime}(x)=f_{2}(k-x) f_{1}(x)\left[\psi_{1}(x)-\psi_{2}(k-x)\right]
$$

Given the definition of $\psi_{i}(\cdot), B^{\prime}\left(k-\underline{v}_{2}\right)>0$ and $B^{\prime}\left(\underline{v}_{1}\right)<0$. From Lemma 5 , $\psi_{i}(\cdot)$ is a decreasing function provided $F_{i}(\cdot)$ is a regular distribution. Therefore $B^{\prime}(\cdot)$ decreases from a positive value at $k-\underline{v}_{2}$ to a negative value at $\underline{v}_{1}$. Therefore, there is an interior $x^{*}$ at which $B^{\prime}\left(x^{*}\right)=0$ and is the maximum of $B(\cdot)$.

Proof of Proposition 7. Let $\mathcal{E}$ be an equilibrium strategy profile and let $x_{i} \leq v_{i}$ be the cutoff such that Player $i$ reports $y$ if and only if her type $v_{i} \geq x_{i}$. Let $\hat{F}_{i}$ be the posterior of Player $i$ when following an announcement $n$ which is truncation of $F_{i}$ to $\left[\underline{v}_{i}, x_{i}\right]$. Let $\tilde{F}_{i}$ be the posterior of Player $i$ when following an announcement $y$ which is truncation of $F_{i}$ to $\left[x_{i}, \bar{v}_{i}\right]$. Note that $\hat{F}_{i}$ and $\tilde{F}_{i}$ are both concave given that $F_{i}$ is concave to begin with. Proposition 2 applies in each continuation game following the announcements. The rest of the proof uses this.

With the posteriors as above, the total benefit of the lowest possible types following announcement's $(n, n),(y, n),(n, y)$ and $(y, y)$ are respectively $\underline{v}_{1}+\underline{v}_{2}, x_{1}+\underline{v}_{2}, \underline{v}_{1}+x_{2}$ and $x_{1}+x_{2}$. Given our hypotheses, in the first three cases the sum is less than $k$. Therefore by Proposition 2, 0-equilibrium is the unique equilibrium and if at all the project is completed, it must be at the node $(y, y)$.

If $x_{1}+x_{2}<k$, the project cannot be completed at the $(y, y)$ node either and the dominance of $\mathcal{E}$ by some simple equilibrium is immediate. Assume then that $x_{1}+x_{2} \geq k$.

Let $q_{i}\left(v_{i}\right)$ denote the probability that the project is completed conditional on the history being $(y, y)$. Then using well known results for incentive compatible mechanisms, we have

$$
U_{i}^{\mathcal{E}}\left(v_{i}\right)= \begin{cases}0 & \text { if } v_{i} \leq x_{i} \\ \left(1-F_{j}\left(x_{j}\right)\right) \int_{x_{i}}^{v_{i}} q_{i}^{\mathcal{E}}(t) d t & \text { if } v_{i} \geq x_{i}\end{cases}
$$

Now let $x=x_{1}$ and consider $\mathcal{S}\left(x_{1}\right)$.

An inspection of the formulas 14, 11) and 12 shows that $U_{i}^{\mathcal{S}(x)}\left(v_{i}\right) \geq U_{i}^{\mathcal{S}}\left(v_{i}\right)$ for all types $v_{i}$. The inequality is strict for the types $v_{2} \in\left[x_{2}, k-x_{1}\right]$ whenever $x_{1}+x_{2}>k$.

Let us now turn to Pareto dominance of equilibria in terms of ex-ante payoffs. This must be a subset of the interim-efficient equilibria, which are the simple strategies. Recall that for this part of the Proposition, we assume that $\left(F_{1}, F_{2}\right)$ are regular. The ex-ante payoffs in $\mathcal{S}(x)$ are given by the formulas in 13 .

Let $x_{i}^{*}$ be the maximum of $W_{i}(\cdot)$ in the region $\left[k-\bar{v}_{2}, \bar{v}_{1}\right]$. If we show that $x_{i}^{*}$ is unique and that $x_{1}<x_{2}$, it would then follow that only those $\mathcal{S}(x)$ for for $x \in\left[x_{1}^{*}, x_{2}^{*}\right]$ are ex-ante 
efficient. Toward this end, first note that

$$
W_{1}^{\prime}(x)=f_{2}(k-x) G_{1}(x)\left[\frac{1}{G_{1}(x)} \int_{x}^{\bar{v}_{1}} G_{1}(t)-\frac{G_{2}(k-x)}{f_{2}(k-x)}\right]
$$

The sign of $W^{\prime}(x)$ depends on the difference between the two expressions in the square brackets. The value of the second expression is zero when $x=k-\bar{v}_{2}$, and since $F_{2}$ is regular, it is increasing to the right of $k-\bar{v}_{2}$. Differentiate the first term and use Eq. (10) note that the first term is decreasing in $x$. Its value is positive at $x=\underline{v}_{1}$, which is to the left of $k-\bar{v}_{2}$ (by admissibility) only falls to zero at $x=\bar{v}_{1}$, which is to the right of $k-\bar{v}_{2}$. Therefore, these two have a unique intersection $x_{1}^{*}$. To the left of $x_{1}^{*}, W_{1}^{\prime}$ is positive and to its right $W_{1}^{\prime}$ is negative. Therefore $x_{1}^{*}$ is the unique maximum of $W_{1}(\cdot)$. Similarly, $W_{2}(\cdot)$ also has a unique maximum, $x_{2}^{*}$.

To prove that $x_{1}^{*}<x_{2}^{*}$, use 10 to note that

$$
W_{1}^{\prime}(x)<f_{2}(k-x) G_{1}(x)\left[\frac{G_{1}(x)}{f_{1}(x)}-\frac{G_{2}(k-x)}{f_{2}(k-x)}\right]
$$

Now let $x^{*}$ denote the unique maximum of the expression $G_{2}(k-x) G_{1}(x)$ when $x$ lies in $\left[k-\bar{v}_{2}, \bar{v}_{1}\right]$. It may be verified that $x^{*}$ is well defined due to the fact that $F_{1}$ and $F_{2}$ are regular. It may also be verified that whenever $x>x^{*}$, the expression in the square brackets of (15) is negative. Therefore whenever $x \geq x^{*}, W_{1}^{\prime}(x)<x$ is negative, which implies that $x_{1}^{*}<x^{*}$. A similar argument shows that $x_{2}^{*}>x^{*}$ and hence $x_{1}^{*}<x_{2}^{*}$.

\section{Proof of Proposition 8. Consider any $\mu \in \mathcal{M}$.}

Integrating by parts the expression for interim utility given in Lemma 6 , we have the following well known expressions for $U_{i}$, the ex-ante utility and $x_{i}$, the expected contribution ex-ante:

$$
\begin{aligned}
U_{i} & =E_{\mathrm{v}}\left[\frac{1-F_{i}\left(v_{i}\right)}{f_{i}\left(v_{i}\right)} p(\mathrm{v})\right]+U_{i}\left(\underline{v}_{i}\right) \\
x_{i} & =E_{\mathrm{v}}\left[\left(v_{i}-\frac{1-F_{i}\left(v_{i}\right)}{f_{i}\left(v_{i}\right)}\right) p(\mathrm{v})\right]-U_{1}\left(\underline{v}_{1}\right) \\
q_{i}\left(\hat{v}_{i}\right) & \geq q_{i}\left(v_{i}\right) \text { whenever } \hat{v}_{i} \geq v_{i}
\end{aligned}
$$

Let

$$
\Gamma(p)=E_{\mathrm{v}}\left[\left(v_{1}-\frac{1-F_{1}\left(v_{1}\right)}{f_{1}\left(v_{1}\right)}+v_{2}-\frac{1-F_{2}\left(v_{2}\right)}{f_{2}\left(v_{2}\right)}-k\right) p(\mathrm{v})\right]
$$

and write the ex-ante budget balance condition $x_{1}+x_{2}-E(p(\mathrm{v})) k \geq 0$ as

$$
\Gamma(p) \geq U_{1}\left(\underline{v}_{1}\right)+U_{2}\left(\underline{v}_{2}\right)
$$

Since the interim utility must be non-decreasing in a player's type, interim individual rationality is implied by

$$
U_{i}\left(\underline{v}_{1}\right) \geq 0 \quad \text { for } i=1,2
$$


Given $\lambda \in[0,1]$, consider the programming problem $\mathbf{P}_{\lambda}$ defined below:

$$
\begin{array}{rll}
\mathbf{P}_{\lambda}:=\max _{q(\mathrm{v})} \lambda U_{1}+(1-\lambda) U_{2} & \text { s.t. } & \text { i). } \quad \Gamma(p) \geq U_{1}\left(\underline{v}_{1}\right)+U_{2}\left(\underline{v}_{2}\right) \\
& \text { ii). } \quad U_{i}\left(\underline{v}_{i}\right) \geq 0 \quad \text { for } i=1,2 .
\end{array}
$$

Observe that $U_{i}$ involves the expectation of a term that is always non-negative. Therefore, any solution of $\mathbf{P}_{\lambda}$, inequality (i) must be binding. Consequently, $\mathbf{P}_{\lambda}$ is isomorphic to the less constrained problem described on page 160, Williams (1987). To see this, intuitively regard Player 1 as seller whose cost is $\hat{v}_{1}=k-v_{1}$. With this change of variable, (18) can be rewritten as

$$
\Gamma(p)=E_{\left(\hat{v}_{1}, v_{2}\right)}\left[\left(v_{2}+\frac{F_{2}\left(v_{2}\right)-1}{f_{2}\left(v_{2}\right)}-\hat{v}_{1}-\frac{\hat{F}_{1}\left(\hat{v}_{1}\right)}{\hat{f}\left(\hat{v}_{1}\right)}\right) p(\mathrm{v})\right]
$$

which is exactly eq. (4) in Williams (1987). Continuing the analogy of a buyer and seller, the cost of the seller varies between, using the notation of Williams (1987), $a_{1}=k-\bar{v}$ and $b_{1}=k-\underline{v}$ while the buyer's value ranges between $a_{2}=\underline{v}$ and $b_{2}=\bar{v}$. Since $a_{1} \geq a_{2}$ and $b_{1} \geq b_{2}$ and $F_{1}, F_{2}$ are regular 10 , Theorem 4 of Williams (1987), which is based entirely on solutions to $\mathbf{P}_{\lambda}$, applies. Any ex-ante efficient allocation of $\mathcal{M}$ involves a $p$ such that $p(\mathrm{v})=1$ if $\mathrm{v}$ lies above a line $\varphi\left(v_{1}\right)=m v_{1}+c$, and $\Gamma(p)=0$. In addition, $p$ is ex-post individually rational. Letting $x_{0}$ and $y_{0}$ denote the points of intersection of this line with $v_{2}=\bar{v}$ and $v_{1}=\bar{v}$ respectively, we note that $k-\bar{v} \leq x_{0}, y_{0}<\bar{v}$, since $p(\cdot)$ is ex-post individually rational. Substituting for $m=-\left(\bar{v}-y_{0}\right) /\left(\bar{v}-x_{0}\right)$ and $c=\bar{v}-m x_{0}$, an explicit calculation gives

$$
\Gamma(p)=\frac{1}{12}\left(\bar{v}-x_{0}\right)\left(\bar{v}-y_{0}\right)\left(x_{0}+y_{0}+\bar{v}-3 k / 2\right) .
$$

Writing $g(\alpha)=3 k / 2-\bar{v}-\alpha$, we note that the pairs $\left(x_{0}, y_{0}\right)=(\alpha, g(\alpha))$ where $\alpha \in[k-\bar{v}, k / 2]$, are the solutions to the equation $\Gamma(p)=0$. This proves that every $p \in \mathcal{P}$ corresponds to $p^{\alpha}(\cdot)$ in which the project is undertaken if and only if $\mathrm{v}$ lies above the line joining $(\alpha, \bar{v})$ and $(\bar{v}, g(\alpha))$. This proves Part 1.

To prove Part 2, we begin by checking the optimality of players' responses at the contribution stage. It is enough to check that at every node, the actions constitute a Nash equilibrium. This is clear at nodes where no contributions are made since zero contributions, by (1), are always an equilibrium.

Consider then a node $\mathrm{v}$ such that $v_{2} \geq m_{\alpha} v_{1}+c_{\alpha}$. The payoff of Player 1 is $v_{1}-x_{1}\left(v_{1}, v_{2}\right)$. We will now verify this is non-negative. To see this, first note that $m_{\alpha}$ ranges from $-1 / 2$ to -2 and therefore $a_{2} \leq 0$ and $a_{1} \geq 0$. Use the facts that $\bar{v}=m_{\alpha} \alpha+c_{\alpha}$ (since $(\alpha, \bar{v})$ lies on the boundary line) and $2 a_{1}+a_{2} m_{\alpha}=1$ to conclude the following sequence of (in)equalities.

$$
\begin{aligned}
v_{1}- & x_{1}\left(v_{1}, v_{2}\right) \\
& =\left(1-a_{1}\right) v_{1}-a_{2} v_{2}-a_{3} \\
& \geq\left(1-a_{1}-a_{2} m_{\alpha}\right) v_{1}-a_{2} c_{\alpha}-\alpha / 2+\left(\bar{v}+c_{\alpha}\right) / 2 \quad\left(\text { since } v_{2} \geq m_{\alpha} v_{1}+c_{\alpha}\right) \\
& =a_{1} v_{1}-a_{2} c_{\alpha}-\alpha / 2+a_{2}\left(m_{\alpha} \alpha+2 c_{\alpha}\right) / 2 \\
& =a_{1} v_{1}-\left(1-m_{\alpha} a_{2}\right) \alpha / 2=a_{1}\left(v_{1}-\alpha\right) \geq 0
\end{aligned}
$$

\footnotetext{
${ }^{10}$ Recall $F_{i}$ is uniform and is hence regular.
} 
A similar calculation shows that $v_{2}-x_{2}\left(v_{1}, v_{2}\right) \geq 0$. Therefore $\left(x_{1}\left(v_{1}, v_{2}\right), x_{2}\left(v_{1}, v_{2}\right)\right)$ constitutes a Nash equilibrium of the complete information contribution game at the node v.

It remains to check that the strategies are incentive compatible at the communication stage. Note that the marginal probability of completion if Player 1 reports $v_{1}$ is $q_{1}\left(v_{1}\right)=$ $\left(1-F\left(m_{\alpha} v_{1}+c_{\alpha}\right)\right)$ when $v_{1} \geq \alpha$ and $q_{1}\left(v_{1}\right)=0$ otherwise. Likewise for Player $2, q_{2}\left(v_{2}\right)=$ $\left(1-F\left(\left(v_{2}-c_{\alpha}\right) / m_{\alpha}\right)\right)$ if $v_{2} \geq g(\alpha)$ and $q_{2}\left(v_{2}\right)=0$ otherwise. Through a direct computation, it may be verified that

$$
E_{v_{j}}\left[x_{i}\left(v_{i}, v_{j}\right)\right]=v_{i} q_{i}\left(v_{i}\right)-\int_{\underline{v}}^{v} q_{i}(t) \mathrm{dt}
$$

Indeed, the coefficients $a_{1}, a_{2}$ and $a_{3}$ were obtained by conjecturing that $x_{i}(\cdot, \cdot)$ is linear and matching the coefficients on either side of the above equation. The above is of course the well known necessary condition that is implied by any incentive compatible mechanism on interim payments. It is also well known (and can be verified directly) that it is a sufficient condition for truthful reporting provided $q_{1}(\cdot)$ and $q_{2}(\cdot)$ are respectively increasing for $v_{1} \geq \alpha$ and $v_{2} \geq g(\alpha)$. This clearly holds here since $m_{\alpha}<0$.

\section{REFERENCES}

Sandeep Baliga and Stephen Morris. Co-ordination, spillovers, and cheap talk. Journal of Economic Theory, 105(2):450-68, August 2002.

Sandeep Baliga and Tomas Sjostrom. Arms race and negotiations. Review of Economic Studes, 71(2):351-69, 2003.

Vincent P Crawford and Joel Sobel. Strategic information transmission. Econometrica, 50 (6):1431-51, November 1982.

Ulrich Doraszelski, Dino Gerardi, and Francesco Squintani. Communication and voting with double-sided information. Contributions to Theoretical Economics, 1(3), 2003.

Joseph Farrell and Robert Gibbons. Cheap talk can matter in bargaining. Journal of Economic Theory, 48(1):221-37, June 1989.

Joseph Farrell and Matthew Rabin. Cheap talk. Journal of Economic Perspectives, 10(3): 103-18, Summer 1996.

Francoise Forges. Equilibria with communication in a job market example. Quarterly Journal of Economics, 105(2):375-98, May 1990.

Drew Fudenberg and Jean Tirole. Game Theory. Cambridge, Mass. and London: MIT Press, 1991.

Steven A Matthews and Andrew Postlewaite. Pre-play communication in two-person sealedbid double auctions. Journal of Economic Theory, 48(1):238-63, June 1989.

Flavio M Menezes, Paulo K Monteiro, and Akram Temimi. Private provision of discrete public goods with incomplete information. Journal of Mathematical Economics, 35(4):493-514, July 2001. 
Roger Myerson. Optimal auctions. Mathematics of Operations Research, 6:58-63, 1981.

Mark A Satterthwaite and Steven R Williams. Bilateral trade with the sealed bid k-double auction: Existence and efficiency. Journal of Economic Theory, 48(1):107-33, June 1989.

Steven R Williams. Efficient performance in two agent bargaining. Journal of Economic Theory, 41(1):154-72, February 1987. 